\title{
A Notch-Wire Composite Antenna for Polarization Diversity Reception
}

\author{
Nobuhiro Kuga, Associate Member, IEEE, Hiroyuki Arai, Member, IEEE, and Naohisa Goto, Fellow, IEEE
}

\begin{abstract}
This paper presents a notch-wire composite antenna for polarization diversity reception in an indoor base-station system. A three-notched disk antenna and a wire antenna are proposed as component antennas for the horizontal and the vertical polarization, respectively. These component antennas are unified as a single composite diversity antenna by mounting the wire antenna on the notched disk. Antenna characteristics are calculated using the method of moments (MoM) with wire grid models and examined in terms of component arrangement and terminal isolation. It is found that maximum isolation is obtained when the current path of the wire antenna is where the current exciting the $H$-pol element is cancelled. Consequently, both component antennas may work independently even in the composite form. The quasi-monopole pattern is also confirmed for each polarization.
\end{abstract}

Index Terms - Antennas.

\section{INTRODUCTION}

$\mathbf{S}$ EVERAL diversity schemes have been used to obtain a high-channel quality in mobile wireless communications. Space diversity [1] and pattern diversity [2] are very popular in land-mobile communication, but require large mounting space to obtain high-diversity gain. Even concerning omnidirectional antennas, the spacing requirements are generally $0.4 \lambda$ or more. Energy density reception scheme has a potential to be a quite small diversity system because it utilizes the $E$ - and $H$-field components at the same position [3]. A flat antenna based on this theory has been also proposed [4], however, these schemes are effective only in single polarization environment with the Rayleigh fading. On the other hand, the use of two orthogonal polarizations, usually the vertical and the horizontal polarization, allows reduction of the signal fading effect without physical separation of the branches because cross correlation between orthogonal polarization components is small in a multipath environment [5]. In addition, it may also reduce the polarization mismatching on the line of sight. These are quite attractive properties for indoor wireless networks including portable handsets. The effectiveness of the scheme was also reported through the experimental evaluation at 1800 $\mathrm{MHz}$ [6].

Manuscript received February 28, 1997; revised January 9, 1998

N. Kuga is with the Antenna Development Project, 3rd Division of Optoelectronic System Laboratory, Takasago Works, Hitachi Cable, Ltd., Hitachi-shi, 3191414, Japan.

H. Arai is with the Faculty of Engineering, Yokohama National University, Yokohama-shi, 2400067 Japan.

N. Goto is with the Faculty of Engineering, Takushoku University, Hachiojishi, 1930944 Japan.

Publisher Item Identifier S 0018-926X(98)04312-9.
A base-station antenna mounted on a ceiling for an indoor wireless network requires a constant radiation pattern in the horizontal plane and a null along the vertical axis as a quarter wavelength monopole antenna. This permits high mobility of remote terminals. A low-profile configuration is also required from an aesthetic view point. A polarization diversity antenna is easily realized using a patch antenna with two orthogonal feeding [7], however, such a method cannot be applicable to the indoor base-station antenna because it is difficult to obtain the horizontal pattern, which is omnidirectional for both dual polarizations.

This paper presents a composite notch-wire antenna for polarization diversity reception in an indoor base-station system. A three-notched disk antenna and a wire-loop antenna are proposed as the component antennas for the horizontal polarization and the vertical polarization, respectively. Their characteristics are examined using the method of moments (MoM) with thin-wire approximations that are verified through measurement. Current distribution on the component antennas are also calculated. A composite polarization diversity antenna, which consists of the disk- and wire-type component antennas is also proposed. Antenna characteristics are examined in terms of antenna configuration and terminal isolation. It is found that maximum isolation is obtained when the current path of the wire antenna is where the current exciting the $H$-pol wave is cancelled. Both component antennas may work independently, even in the composite form, therefore, the quasi-monopole pattern is also obtained for each polarization.

\section{COMPONEnT AnTEnNAS}

First, two antennas are proposed as component antennas for polarization diversity reception. Fig. 1 shows a disk antenna for the horizontal polarization reception ( $H$-pol component antenna) consisting of a circular disk loading three notches spaced $120^{\circ}$ apart. Outer radius of the disk is $a_{h}$ and each notch has angular width $\alpha$, and depth $l_{d}$. The phase and amplitude exciting three notches are equal. A feed port is placed at near the center of the notched disk with a threebranch power divider to deliver the power. In the following, a divider employing three coaxial lines with a characteristic impedance $Z_{0}$ and a length $L$ was used of which the outer conductor is attached to the notched disk and the inner conductor is connected to the feed probe. The antenna can be also fabricated on a dielectric substrate using microstrip lines. An antenna with a similar structure was proposed as a energy density reception antenna [3], but it differs significantly in the feeding phase and polarization characteristics. The energy 


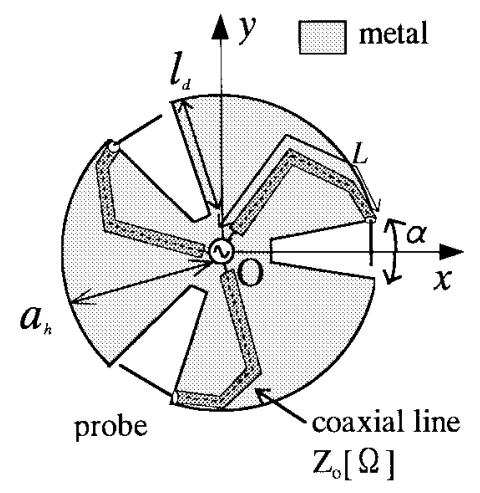

Fig. 1. Three-notched disk antenna for the $H$-pol reception.

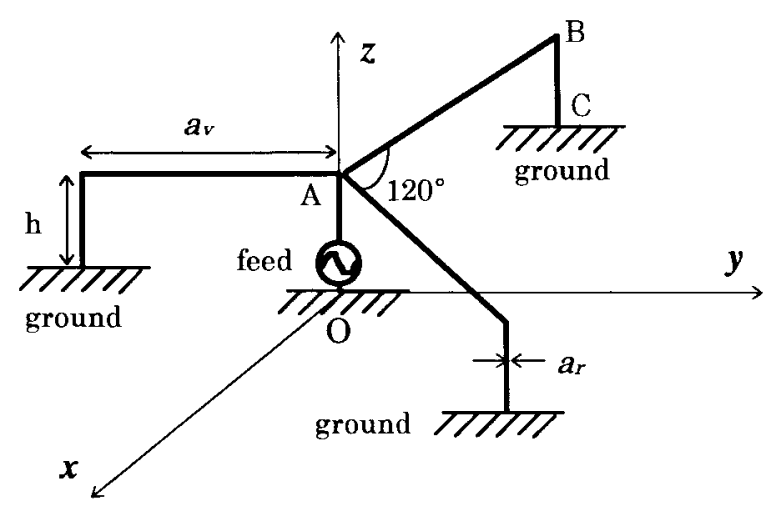

Fig. 2. Wire antenna for the $V$-pol reception.

density reception scheme utilizes fields with $90^{\circ}$ of phase difference and single polarization.

Next a wire antenna for the vertical polarization reception is shown in Fig. 2. It consists of three vertical wire-loop with $120^{\circ}$ rotation and the power is supplied at the vertical wire placing at the rotation center. Each loop has a horizontal wire with length $a_{h}$ and vertical wire with height $h$. The perimeter of a component loop (OABC) is about a half wavelength and has a ratio of $\mathrm{OA}: \mathrm{AB}=1: 5$.

Next, characteristics of the component antennas are described. They were calculated using the MoM with the wire grid model and using numerical electromagnetic code 2 NEC2 [8]. Each wire is divided into short segment of which the length is less than $\lambda / 20$, and $\delta$-gap voltage source is implied on the feed segment. The input impedance of the $V$-pol wire antenna is calculated assuming an infinite ground plane and measured using an large but finite size of ground plane. The $H$-pol element is modeled as a wire-grid model with three voltage sources without the power divider. No ground plane is assumed for the $H$-pol antenna. Using this model, a driving admittance of the feeding probe of the notch antenna is calculated. In next step, the probe admittance is obtained by transforming to terminal admittance via a transmission line with a characteristic impedance $Z_{0}$ and a length $L$. Input admittance of the $H$-pol antenna is obtained in a form of summation of the terminal admittance. In this section, the component antenna tests were performed separately.

Fig. 3(a) shows the input impedance of the $H$-pol component antenna, where the solid line and dotted line represent

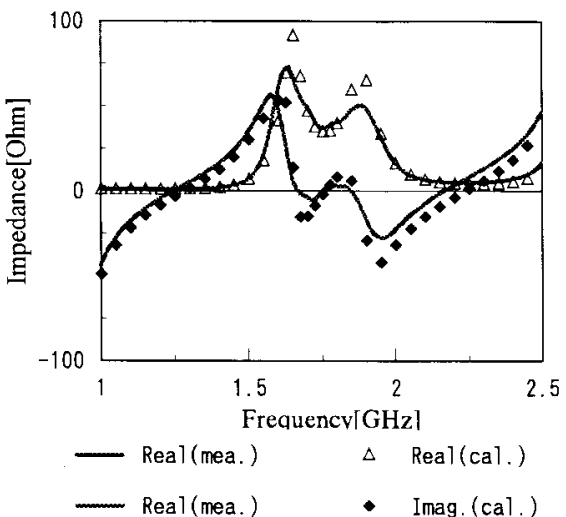

$$
a_{h}=50, l_{d}=35, L=110[\mathrm{~mm}], \alpha=30^{\circ}, Z_{0}=75[\Omega]
$$

(a)
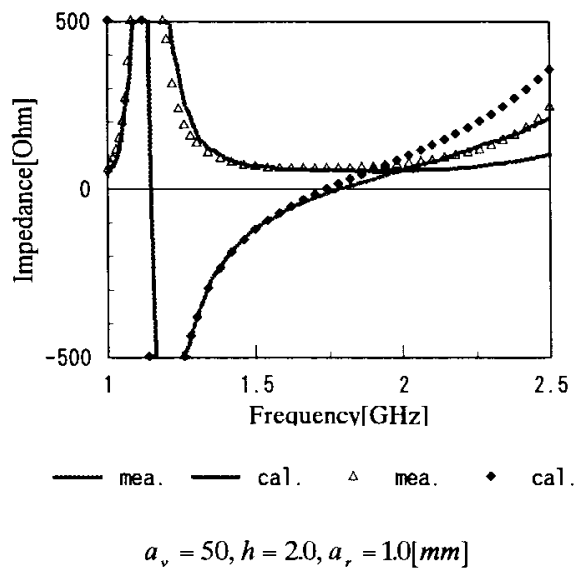

(b)

Fig. 3. Input impedance characteristics of the component antennas. (a) Notched disk antenna (H-pol, no mounting plate). (b) Wire antenna (V-pol, infinite ground plane).

measurement and calculation. The disk radius $a=50 \mathrm{~mm}$, the notch width $\alpha=30^{\circ}$, and the characteristic impedance of coaxial line in the power divider $Z_{0}$ is $75 \Omega$. Good agreement is obtained and validity of the wire-grid model is confirmed. As the power divider works as an impedancematching network, enhancement of the impedance bandwidth is also obtained in the form of dual resonance [9]. Therefore, the fractional bandwidth (return loss $<-10 \mathrm{~dB}$ ) of the $\mathrm{H}$ pol antenna is $17 \%$ for a center frequency of $1763 \mathrm{MHz}$. Fig. 3(b) shows the input impedance characteristics of the $V$-pol component antenna. Good agreement is also obtained between calculation and measurement. In the experiment, the antenna is composed of enamel coated wires with a radius of $0.5 \mathrm{~mm}$ and a circular ground plane with a radius of 300 $\mathrm{mm}$. The fractional bandwidth of the $V$-pol element is about $13 \%$ for a center frequency of $1788 \mathrm{MHz}$, which is narrower than one of the notched-disk antenna because of no matching network. When designing the composite antenna, its operation frequency depends on the $V$-pol element because the $H$-pol element has the wide-band characteristic.

Next, the current distributions by the MoM calculation are shown. One of the $H$-pol antennas is shown in Fig. 4, where (a) and (b) are distributions at dual-resonance frequencies. The 


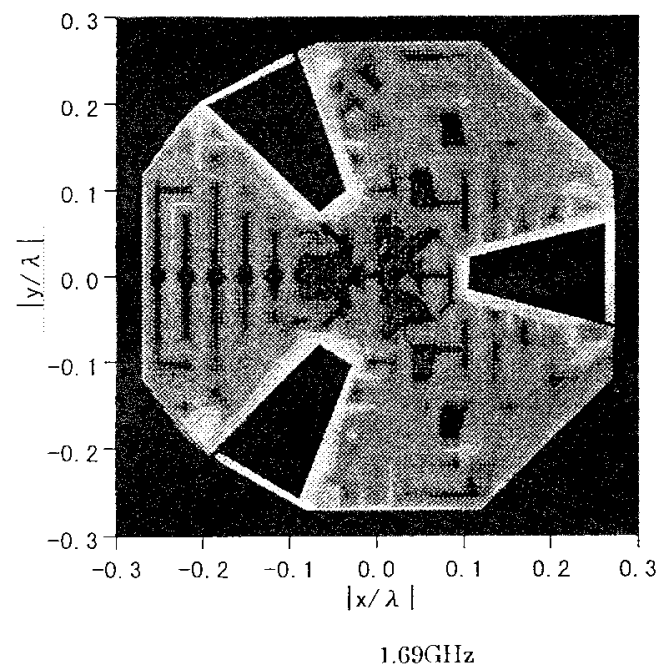

(a)
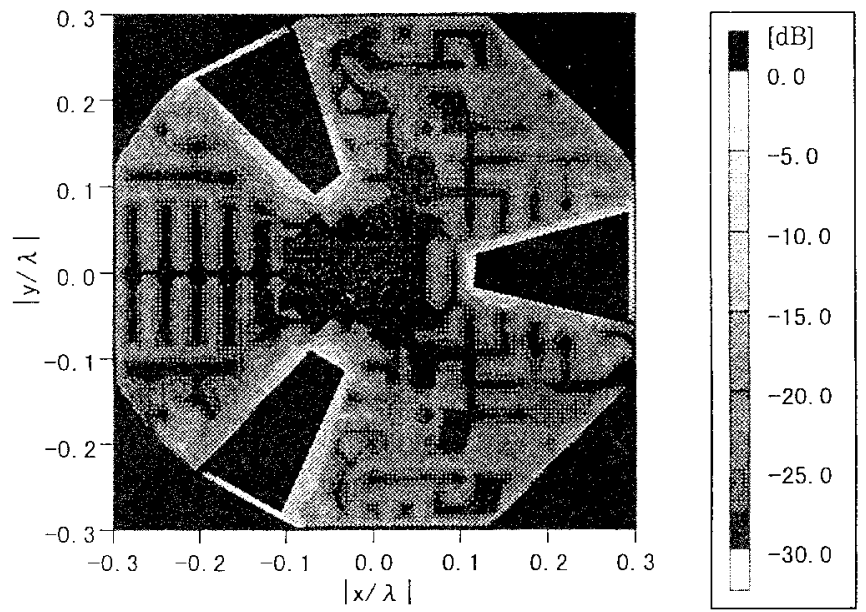

(b)

Fig. 4. Current distribution on the notched-disk component antenna. (a) 1.69 GHz. (b) $1.85 \mathrm{GHz}$.

calculated amplitude is larger in Fig. 4(a) than in Fig. 4(b), therefore, all the data are normalized by the maximum diskcurrent amplitude at $1.69 \mathrm{GHz}$. A common phenomena is that the current excited by the feeding probe flows along the notch and is cancelled near the disk center. Fig. 5 shows the current distribution along the $V$-pol wire antenna. On the $V$ pol element, since the phase of the current is inverted at the distance of $a_{v} / \lambda=0.1$, a current in phase with the one of the center wire flows on the outer wires with a relative amplitude of $-3.5 \mathrm{~dB}(0.67)$. Current on the horizontal portion does not contribute to radiation; only the vertical portion contributes to radiation, therefore, the asymmetry of the horizontal pattern is smaller than the one of the $H$-pol.

\section{Composite Polarization Diversity ANTEnNa}

Ideally, in the polarization diversity reception, the branches can be placed in the same place because uncorrelated fields are received regardless of their arrangement. Therefore, combining the two-component antennas, we propose a composite antenna for two-branch polarization diversity reception. Its configura-

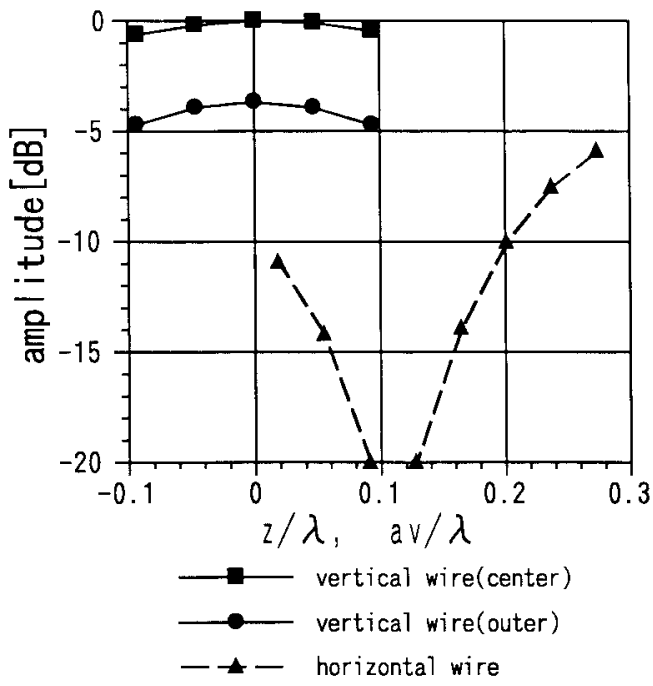

Fig. 5. Current distribution of the wire antenna.

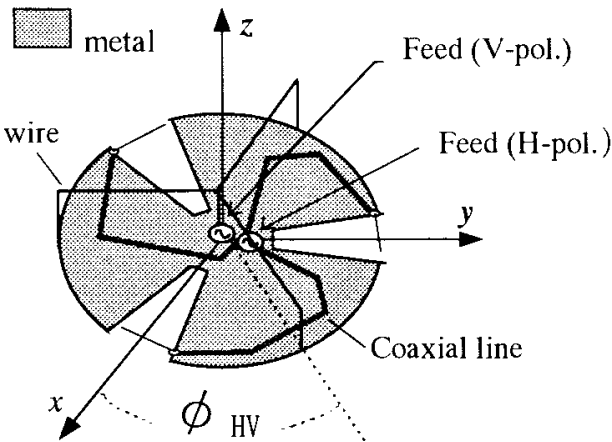

Fig. 6. Composite antenna for two-branch polarization diversity reception.

tion is shown in Fig. 6. A conducting mounting plate may be used to stabilize antenna performance, however, it is not used in this study. The antenna diameter is about $0.6 \lambda$, which is larger than one of a conventional printed dipole, however, the height of $0.12 \lambda$ is lower and more advantageous for indoor use.

The input characteristic of the composite antenna is shown in Fig. 7. Good agreement obtained between calculation and measurement shows validity of the presented model. The resonant frequency of the component antenna is not changed by the unification, however, the bandwidth of the $V$-pol element is reduced to $8.5 \%$. The bandwidth of $H$-pol element remains wide- and dual-resonance characteristics. Mutual coupling between the feed ports is less than $-10 \mathrm{~dB}$ over the operating bandwidth, as shown in the figure. It should be noted that the coupling is larger in the lower frequency than in higher one. This phenomenon is due to the current distribution, as shown in Fig. 4. In the lower frequency, the $H$-pol current is distributed more along the $V$-pol current path, therefore, the coupling increases.

Here, we examine the element arrangement in terms of branch isolation. Fig. 8 shows an isolation characteristic between the feed ports of the composite antenna, which is calculated versus arrangement angle $\phi_{H V}$ in Fig. 6. Maximum values are obtained when $\phi_{H V}=606^{\circ}, 180^{\circ}$, and $300^{\circ}$ 


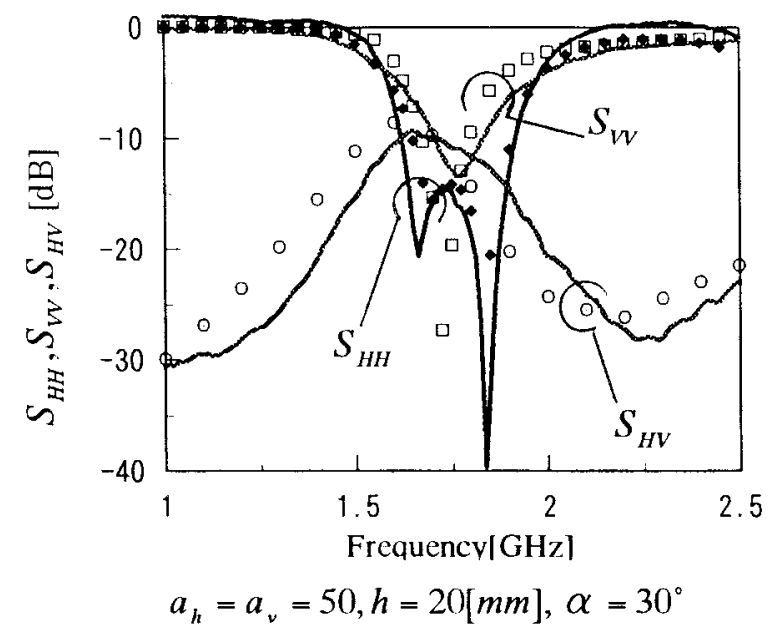

Fig. 7. Input characteristics of the composite polarization diversity antenna as a function of frequency. $S_{V V}, S_{H H}$ are reflection characteristics of $V$-pol and $H$-pol ports, respectively. $S_{H V}$ is the mutual coupling between them.

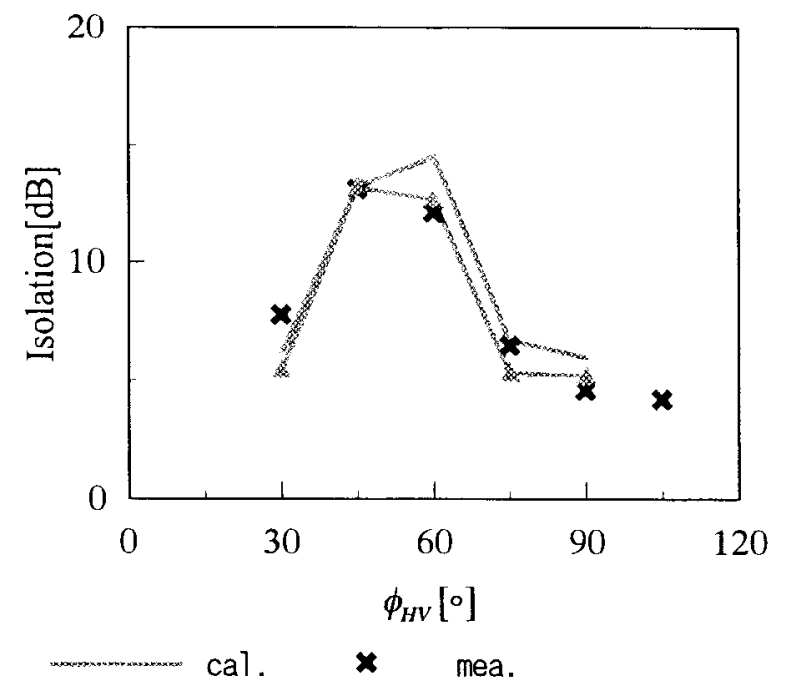

Fig. 8. Branch isolation characteristics as a function of the arrangement angle $\phi_{H V}$.

because on the notched disk, the current path of the $V$-pol element is on where the current exciting the $H$-pol element is cancelled. In the figure, the maximum value is $14 \mathrm{~dB}$ when the notch width $\alpha=30^{\circ}$.

Next, radiation pattern of the composite antenna is shown in Fig. 9. Each pattern represents a co-polarization of the corresponding branch. A quasi-uniform pattern with null in the $z$-axis direction is obtained for both polarizations. Average antenna gain is $0 \mathrm{dBi}$ in the $x y$ plane, however, there exists the degradation within $\pm 2.5 \mathrm{~dB}$ and $\pm 1.5 \mathrm{~dB}$ for the $H$-pol and the $V$-pol pattern, respectively. It is found that the antenna unification increases the horizontal pattern degradation, especially for the $H$-pol antenna.

\section{CONCLUSION}

A notch-wire composite antenna was presented as a polarization diversity antenna. The component antennas were examined using the MoM and their input impedance char-

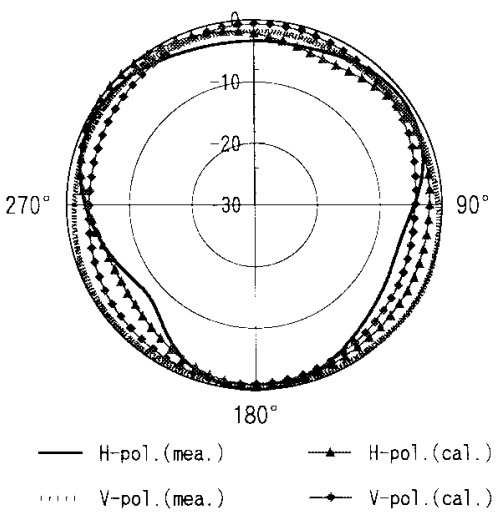

(a)

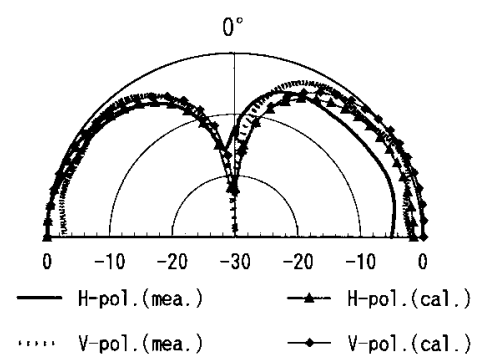

(b)

Fig. 9. Radiation pattern of the composite antenna (measurement at 1.87 $\mathrm{GHz}$ ). (a) $x y$ plane. (b) $z x$ plane.

acteristics and current distributions were demonstrated. While the notched-disk antenna for the $H$-pol reception has a wide bandwidth due to the matching network, the wire antenna for the $V$-pol reception does not because of the no-matching network.

Characteristics of the composite polarization diversity antenna were also examined using the MoM and their results are confirmed by experiment. After unification of the component antennas, the bandwidth of the $V$-pol antenna decreased to $8.5 \%$, while the one of the $H$-pol antenna remained $17 \%$ of the $-10 \mathrm{~dB}$ fractional bandwidth. The $H$-pol radiation pattern has a degradation of $\pm 2.5 \mathrm{~dB}$ in the horizontal plane, which is large compared with the $V$-pol as $\pm 3.0 \mathrm{~dB}$. Mutual coupling characteristics are also examined in terms of the current distribution and antenna arrangement angle. A field test of the composite diversity antenna is left as a future problem.

\section{REFERENCES}

[1] W. C. Jakes, Jr., "A comparison of specific space diversity techniques for reduction of fast fading in UHF mobile radio systems," IEEE Trans. Veh. Technol., vol. VT-20, pp. 81-92, Nov. 1971.

[2] Y. Yamada, K. Kagoshima, and K. Tsunekawa, "Diversity antennas for base and mobile stations in land mobile communication systems," Trans. IEICE, vol. E74, pp. 3202-3209, Oct. 1991.

[3] E. N. Gilbert, "Energy reception for mobile radio," Bell Syst. Technol. $J .$, vol. 44, no. 8, pp. 1779-1803, Oct. 1965.

[4] S. Hosono, H. Arai, and N. Goto, "Flat two branches diversity antenna," in Proc. IEICE Gen. Conf., Chiba, Japana, Mar. 1992, B-91, pp. 2-91.

[5] M. Nakano, T. Satoh, and H. Arai, "Up-link polarization diversity measurement of hand-held terminal," in IEEE Antennas Propagat. Soc. Dig., pp. 1940-1943, July 1995.

[6] A. Turkmani, A. Arowojolu, P. Jefford, and C. Kellet, "An experimental evaluation of the performance of two-branch space and polarization diversity schemes at $1800 \mathrm{MHz}$," IEEE Trans. Veh. Technol., vol. 44, pp. 318-326, May 1995. 
[7] T. Nara, Y. Ebine, and Y. Yamada, "Characteristics of polarization diversity base station antenna," in Proc. IEICE Nat. Conv., Nigata, Japan, Mar. 1986, no. 643, pp. 3-80.

[8] G. J. Burk and A. J. Poggio, "Numerical electromagnetic code-Method of moment analysis," Lawrence Livermore Laboratory, Livermore, CA, 1981.

[9] H. F. Pues and A. R. Van De. Capelle, "An impedance-matching technique for imcreasing the bandwidth of microstrip antennas," IEEE Trans. Antennas Propagat., vol. 37, pp. 1345-1354, Nov. 1989.

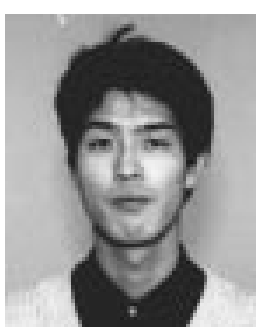

Nobuhiro Kuga (S'94-A'97) received the B.S., M.S., and Ph.D. degrees in engineering from Yokohama National University, Japan, in 1992, 1994, and 1997, respectively.

He currently works as a Researcher of the Optoelectronic System Laboratory, Hitachi Cable. Ltd., Hitachi City, Japan. His current research interests are small antennas and base station antennas for mobile communications.

Dr. Kuga is a member of IEICE Japan.

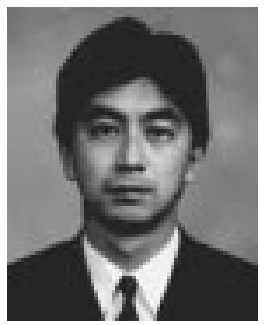

Hiroyuki Arai (S'84-M'87) received the B.E. degree in electrical and electronic engineering and the M.E. and D.E. degrees in physical electronics from the Tokyo Institute of Technology, Japan, in 1982, 1984, and 1987, respectively.

$\mathrm{He}$ is currently an Associate Professor in the Division of Electrical and Computer Engineering, Yokohama National University, Japan, and does consulting for the Japan Atomic Energy Research Institute, Tokai Village, for microwave components in radio frequency heating of tokamak plasma. He also collaborates with several companies for mobile station antennas, cellularbase station antennas, antenna-measurement techniques, indoor/outdoor propagation measurement and simulation, and electromagnetic compatibility and wave absorbers. He is the coauthor and author of three textbooks about electromagnetic waves and holds a U.S. and several Japanese patents for antennas.

Dr. Arai was awarded the Meritorious Award on Radio by the Association of Radio Industries and Businesses in 1997.

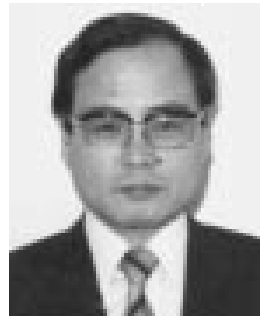

Naohisa Goto (M'68-SM'88-F'91) was born in Utsunomiuya, Japan, on June 8, 1935. He received the B.S., M.S., and D.E. degrees from the Tokyo Institute of Technology, Tokyo, Japan, all in electrical engineering, in 1959, 1961, and 1964, respectively

From 1964 to 1980, he worked as a Research Associate and Associate Professor at the Tokyo Institute of Technology, Japan, as well as an Associate professor at Chiba University, Chiba, Japan. From 1980 to 1996 he was a Professor at the Tokyo Institute of Technology. Since 1996 he has been a Professor at the Takushoku University, Tokyo, Japan. He invented several flat antennas: a radial-line slot antenna; a single-layer slotted waveguide antenna; a self-diplexing ring-patch antenna; and a flat diversity antenna.

Dr. Goto was the general chairman of the 1992 International Symposium on Antennas and Propagation held at Sapporo, Japan. He received an Invention Award from Tokyo Institute of Technology in 1989 for the invention and development of the flat antennas. He also received the Institute of Electronics, Information, and Communication Engineers Achievement Award and Paper Award in 1992 and the Distinguished Award in 1997. 